\title{
Noise-induced inhibitory suppression of frequency-selective stochastic resonance
}

\author{
C. J. Tessone, ${ }^{1}$ E. Ullner, ${ }^{2}$ A. A. Zaikin, ${ }^{3}$ J. Kurths, ${ }^{3}$ and R. Toral ${ }^{1}$ \\ ${ }^{1}$ Institut Mediterrani d'Estudis Avançats (IMEDEA), CSIC-Universitat de les Illes Balears, Ed. Mateu Orfila, Campus UIB, \\ E07122 Palma de Mallorca, Spain \\ ${ }^{2}$ Max-Planck-Institut für Physik komplexer Systeme, Nöthnitzer Straße 38, D01187 Dresden, Germany \\ ${ }^{3}$ Institut für Physik, Universität Potsdam, Am Neuen Palais 10, D14469 Potsdam, Germany \\ (Received 3 August 2005; revised manuscript received 29 May 2006; published 31 October 2006)
}

\begin{abstract}
We study the control of oscillations in a system of inhibitory coupled noisy excitable and oscillatory units. Using dynamical properties of inhibition, we find regimes when the oscillations can be suppressed but the information signal of a certain frequency can be transmitted through the system. The mechanism of this phenomenon is a resonant interplay of noise and the transmission signal provided by certain value of inhibitory coupling. Analyzing a system of three or four oscillators representing neural clusters, we show that this suppression can be effectively controlled by coupling and noise amplitudes.
\end{abstract}

DOI: 10.1103/PhysRevE.74.046220

\section{INTRODUCTION}

Excitable systems are widespread in nature. In many cases, excitability originates from the existence of a bifurcation to an oscillatory state when a control parameter is changed. Prominent examples are some varieties of cells (such as neurons, pancreatic $\beta$-cells, nerve cells from sensitive regions of the body), cardiac tissue, chemical reactions (Belusov-Zavotinsky), etc. (for a comprehensive review on this subject, see Ref. [1]). Most excitable systems require two types of dynamical variables (called generically activator and inhibitor) with different time scales and different influence on the overall behavior of the system. Stochastic effects, in the form of white noise or diversity, are also an important ingredient of the dynamics. The dynamics of oscillatory and excitable systems near the bifurcation has attracted large interest because in this region their sensitivity is greatest and they are suitable for a reliable signal response or information exchange. The study of coherence resonance $[2,3]$ (also named stochastic coherence [4]) and stochastic resonance in nonlinear excitable units [1,5-7] aroused a strong interest in this field.

Of particular interest is the study of coupled excitable systems. Usually, only coupling through the activator variables is considered, leading to a full synchronized dynamics [8]. In this work, we focus our attention on an array of noisy excitable units coupled through inhibitory variables. Such a kind of coupling can be realized, for instance, by a negative coupling constant in an activator variable or by a positive one in an inhibitory variable. Previous work has shown that coupling through the inhibitor variable between identical oscillators may induce many limit cycles of different periods and phase relations $[9,10]$ which are stable in large regions of the control parameter space, a behavior usually referred to as a "dephasing" [11,12] or "phase-repulsive" [13] interaction and which was shown to be a source of multirhythmicity in different systems [14-17]. With noisy elements, a dephasing interaction of stochastic limit cycles (instead of deterministic ones) may result in the coexistence of spatiotemporal regimes selectively sensitive to external signal periods. In such systems, noise plays at least two roles: first, it stimulates firing of stable elements and, thereby, their interaction during return excursions; second, it stimulates transitions be-
PACS number(s): 05.45.-a, 87.19.La, 05.40.Ca, 87.10.+e tween coupling-dependent attractors if the associated lifetime is sufficiently long.

In this paper, we extend our research on the influence of inhibitory coupling. In contrast to our previous investigation of frequency-selective stochastic resonance in linear chains of identical excitable FitzHugh-Nagumo (FHN) models $[18,19]$, we consider chains of nonidentical units and focus on the influence that the internal oscillatory units have on the dynamics of the whole chain. Under the presence of an external signal, we find the counterintuitive result that inhibitory coupling can lead to a multirhythmic state in which the intermediate oscillatory units are in the rest state, while the excitable ones oscillate in synchrony with the signal. This implies that the intermediate units, while "silent," are still able to transmit information along the chain. As discussed at the end of the paper, inhibitory coupling is of relevance for some chemical [20-22] and biological [23] systems and our results point to a generic mechanism of oscillation supression and information transmission. For the sake of simplicity, however, we have only considered a prototype excitable model (the FitzHugh-Nagumo set of equations, also called the Bonhoeffer-van der Pol model [24]). In the next section we present the main result in the simplest case of three FitzHugh-Nagumo units coupled through the inhibitory variable. To extend the study into another architecture, in Sec. III, we analyze an array where two coupled oscillating units are connected from both sides with excitable elements. Our study ends in Sec. IV with some general conclusions and some speculations about possible applications in biological systems.

\section{THREE NONIDENTICAL INHIBITORY COUPLED FITZHUGH-NAGUMO UNITS}

We consider a rather simplistic model with a minimal scheme of connections that can retain the basic structure of the system we want to study: an open, linear chain where both ends have an excitable unit. The middle unit represents an oscillatory unit (Fig. 1). We want to study whether a periodic, subthreshold signal, acting on the left element can reach the right one, in such a way that no oscillations appear in the middle unit. 


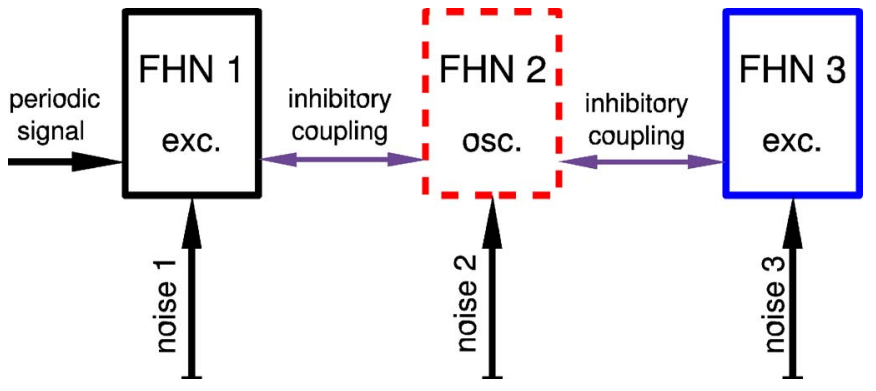

FIG. 1. (Color online) Scheme of the setup for the case $N=3$. At both ends there are excitable units, coupled through inhibitor coupling to the middle (oscillatory) element.

The scheme in Fig. 1 corresponds to three FitzHughNagumo oscillators coupled through the inhibitory variables

$$
\begin{gathered}
\varepsilon \dot{x}_{1}=y_{1}-\frac{x_{1}^{3}}{3}+x_{1}, \\
\dot{y}_{1}=a_{1}-x_{1}+\xi_{1}(t)+A_{s} \cos (\omega t)+D\left(y_{2}-y_{1}\right), \\
\varepsilon \dot{x}_{2}=y_{2}-\frac{x_{2}^{3}}{3}+x_{2}, \\
\dot{y}_{2}=a_{2}-x_{2}+\xi_{2}(t)+D\left(y_{1}-y_{2}\right)+D\left(y_{3}-y_{2}\right), \\
\varepsilon \dot{x}_{3}=y_{3}-\frac{x_{3}^{3}}{3}+x_{3}, \\
\dot{y}_{3}=a_{3}-x_{3}+\xi_{3}(t)+D\left(y_{2}-y_{3}\right),
\end{gathered}
$$

where $\omega=2 \pi / T_{s}$ is the frequency of the input signal with period $T_{s}$. The Gaussian (white) noise sources $\xi_{i}(t)$ satisfy $\left\langle\xi_{i}(t) \xi_{j}\left(t^{\prime}\right)\right\rangle=\sigma_{a}^{2} \delta\left(t-t^{\prime}\right) \delta_{i, j}$.

In a neural context, $x_{i}(t)$ represents the membrane potential of the neuron and $y_{i}(t)$ is related to the time-dependent conductance of the potassium channels in the membrane [25]. The dynamics of the activator variable $x_{i}$ is much faster than that of the inhibitor $y_{i}$, as indicated by the small timescale ratio parameter $\varepsilon$. It is well known that for $\left|a_{i}\right|>1$ a single unit has a stable fixed point and presents excitable behavior: small perturbations are followed by a smooth return to the fixed point, while a perturbation larger than a threshold value induces a return through a large excursion in phase space (a spike). For $\left|a_{i}\right|<1$, the fixed point becomes unstable and a stable limit cycle appears. In this regime, the dynamics consists in a periodic series of spikes. Along this section, we will consider the fixed parameters $\varepsilon=10^{-4}, a_{1}$ $=a_{3}=1.01$, and $a_{2}=0.99$, such that the two end units are excitable and the middle one oscillatory. We have checked that (in the absence of external forcing and noise) the three units retain their excitable or oscillatory character despite the coupling among them, such that the middle unit spikes periodically and the two end units display small subthreshold oscillations around the fixed point.

The issue now is the behavior of these same units when noise and external forcing are present. We will show that it is possible to have a noise-induced regime in which the oscillations of the middle unit are suppressed. To characterize this phenomenon of oscillation suppression, we have computed $[26,27] N_{s}^{(i)}$, the number of spikes per time unit at the $i$ th neuron, defined as the number of times the variable $y_{i}(t)$ surpasses a fixed threshold per time unit. $N_{s}^{(i)}$ represents the reciprocal of the averaged interspike time interval.

An important point is whether in this oscillation suppression regime, noise can help to transmit the information of the subthreshold external signal by a stochastic resonance mechanism. In order to address this issue, we compute the linear response $Q^{(i)}$ of the $i$ th neuron in the chain at the input frequency $\omega[28,29]$ :

$$
Q^{(i)}=\left|\left\langle 2 y_{i}(t) e^{\iota \omega t}\right\rangle\right|
$$

where $\langle\cdots\rangle$ denotes a time average.

\section{A. Oscillation suppression via a noise-induced dynamical trap}

In a previous work [17] it has been shown that in a system of three FitzHugh-Nagumo units in the oscillatory regime (and in the absence of external forcing) the inhibitory coupling leads to two coexisting dynamical attractors, with different natural frequencies. These attractors correspond to an antiphase oscillator movement and to the so-called dynamical trap regime where the middle oscillator is at rest and the two oscillators at the ends oscillate in antiphase. If one now applies a weak external periodic signal to one of the end units and uncorrelated noise to every unit, one can still achieve the suppression of the self-excited oscillations of the middle unit and, at the same time, achieve a reliable transmission of the signal, provided the following two conditions hold: (i) the frequency of the external signal coincides with the natural frequency of the dynamical trap attractor, and (ii) the noise in the system is near the optimal one for the desired signal amplification (i.e., stochastic resonance phenomenon on this attractor).

A similar result appears in our system of three coupled units. For very small noise, the situation is as described at the beginning of the section with the middle unit oscillating and the end units at rest. As noise increases, one observes random switches between this state and a dynamical trap regime in which the middle unit is at rest and the two end units spike in antiphase. This effect can be quantified by measuring the number of spikes $N_{s}^{(i)}$ and the responses $Q^{(i)}$ as a function of the noise intensity. As shown in Fig. 2, one can distinguish several behaviors depending on the period of the external forcing.

(a) This is the case where the period of the input signal equals the natural period of an isolated FitzHugh-Nagumo oscillator ( $T_{s}=2.8$ for $\left.a=0.99\right)$. The noise-induced oscillation suppression described before is apparent in the right panel of this figure, where it is shown that the number of spikes at the middle unit, $N_{s}^{(2)}$, first decreases as the noise intensity increases. This oscillation suppression is maximum at a value of the noise intensity, $\sigma_{a}^{2} \approx 3 \times 10^{-6}$. At noises larger that this value, the number of spikes in the three units are very close to each other. 

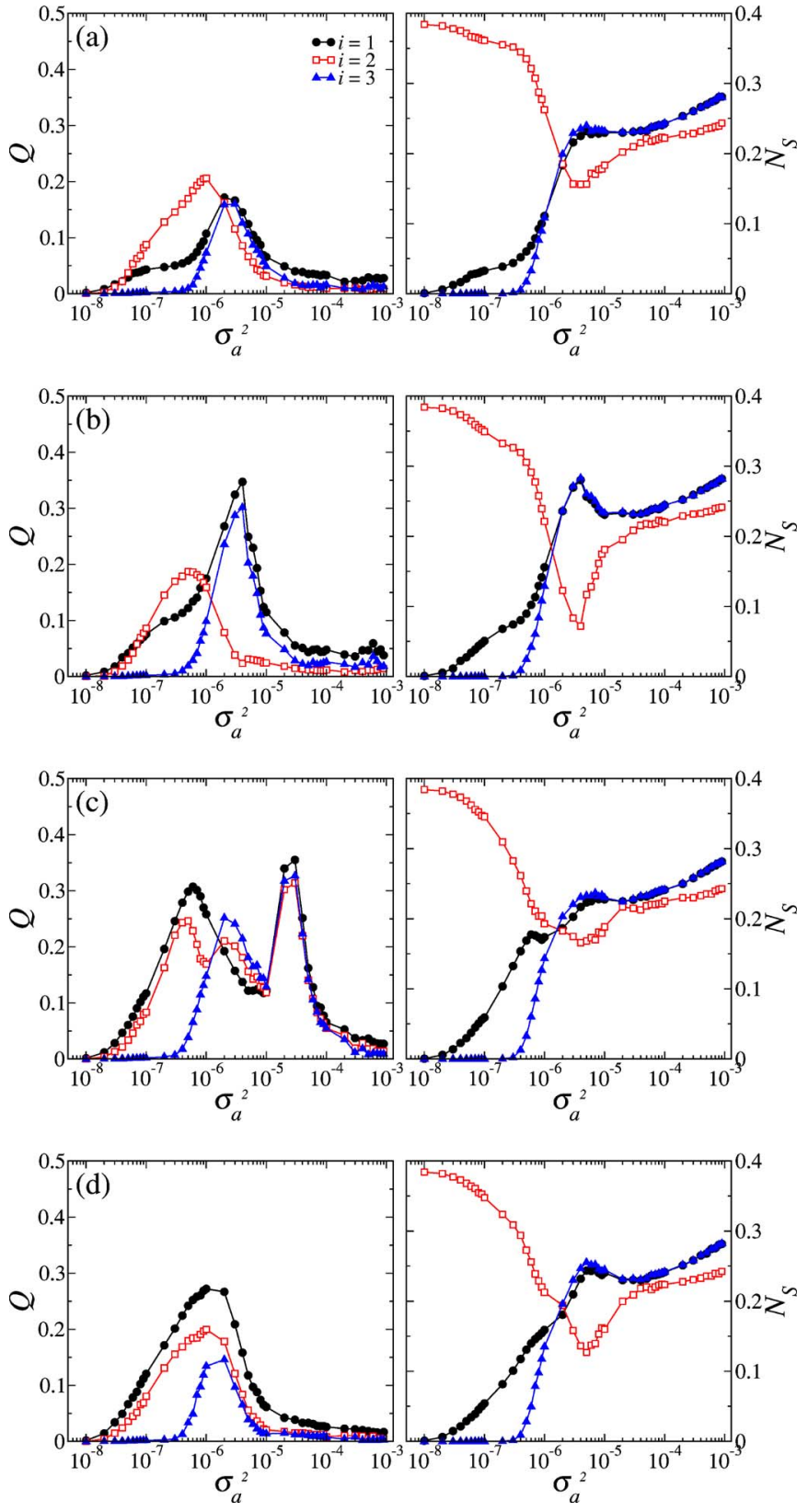

FIG. 2. (Color online) Optimal noise suppresses oscillations while letting the signal (within a certain range) be transmitted. This effect occurs due to dynamical trap, supported by inhibitory coupling. (a) nonresonant, $T_{s}=2.8$; (b) dynamic trap, $T_{s}=3.1$; (c) antiphase resonance, $T_{s}=4.5$; (d) no resonance, $T_{s}=6.0$. Other parameters: $\varepsilon=10^{-4}, a_{1,3}=1.01, a_{2}=0.99, A_{s}=0.01$, and $D=0.15$. The left and right columns correspond to the $Q$ and $N_{s}$ measures.

In the left panel we plot the response $Q^{(i)}$ of each unit. Note that there is a range of values for the noise intensity for which the middle unit responds to the injected signal most effectively than the end units, as signaled by a higher value of the response $Q^{(2)}$. For increasing noise intensity, beyond the value where the oscillation suppression was maximum, all units have a similar response.

(b) For an intermediate range of periods $T_{s} \in[3,3.4]$, we observe that there exists a range of noise intensities $\left(\sigma_{a}^{2}\right.$ $\left.\in\left[10^{-6}, 10^{-5}\right]\right)$ such that the number of spikes is strongly reduced in the middle oscillatory unit, while the response to the driving frequency is better than in the oscillatory unit; i.e., this is the manifestation of the dynamic trap regime. One can clearly see the effective oscillation suppression of the oscillatory middle element [see Fig. 2(b), right] and—despite this suppression in the middle of the chain - the reliable information transport from the first to the last unit by a large linear response $Q$ in these elements [Fig. 2(b), left]. The dynamic trap regime includes an antiphase motion of the first and last units which results in combination with the inhibitory coupling in a suppression of the oscillations of the middle element.

(c) Increasing even further the period, $T_{s}=4.5$, the external signal is now in resonance with, and hence amplifies, the antiphase motion in which the first and last units oscillate in-phase and in antiphase with the middle one. In this case, another interesting regime appears in the noise range $\sigma_{a}^{2}$ $\in\left[10^{-5}, 10^{-4}\right]$ as observed in the right panel of Fig. 2(c), where the spike numbers of all three elements coincide nearly, as well as in the linear response plot (left panel), where all oscillators display a very similar linear response $Q$. This antiphase regime demonstrates a totally different behavior than the dynamic trap regime, case (b) discussed previously. Note that the antiphase regime appears for a much larger noise intensity than the dynamic trap regime, hence showing a double selectivity by the input frequency and the noise intensity.

(d) Finally, for much larger period $T_{s}=6.0$, there is no resonance, Fig. 2(d). This can be observed especially at the linear response $Q$ (left panel) which is much smaller than in the resonant cases. Noteworthy, the last element in the chain exhibits a poor signal response.

\section{B. Control of suppression by the coupling strength}

Noise-induced dynamical trap suppression is possible by the existence of a new attractor originated in the inhibitory nature of the coupling. Hence, the coupling intensity $D$ controls the effectivity of the suppression, as well as the frequency of the attractor. The existence of an optimal value for $D$ is shown in Fig. 3, in which we plot the linear response $Q$ and the spike numbers $N_{s}$ as a function of the coupling intensity. Setting the noise intensity $\sigma_{a}^{2}$ to the value of maximum of the linear response $Q$ [Fig. 2(b), left panel] and varying the strength $D$ of the inhibitory coupling, it is clear the existence of an optimal $D$ such that the middle unit is silent (Fig. 3, right panel), while the first and last units effectively respond to the driving frequency (Fig. 3, left panel).

Since both types of coupling, inhibitory and activator, can be immanent in neural networks, we have investigated how the suppression can be regulated if we tune the coupling from an activator to an inhibitory one. To do this, we have added activator coupling in the model by interchanging $D$ by $(1-\alpha) D$ in the equations for the inhibitory variable $y_{i}$ and inserting the terms $\alpha D\left(x_{2}-x_{1}\right)$ into Eq. (1), $\alpha D\left(x_{1}-x_{2}\right)$ $+\alpha D\left(x_{3}-x_{2}\right)$ into Eq. (2), and $\alpha D\left(x_{2}-x_{3}\right)$ into Eq. (3). These extensions of the model are used only in this section for the calculation of Fig. 4. With help of the new sliding parameter $\alpha$ we change the weight of the type coupling from $\alpha=1$ (pure 


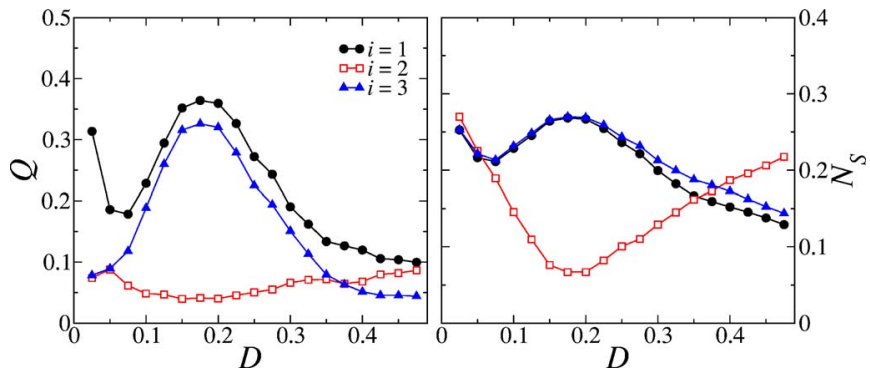

FIG. 3. (Color online) Linear response $Q$ (left) and normalized spike number $N_{s}$ (right) versus inhibitory coupling. The other parameters are $\varepsilon=10^{-4}, a_{1,3}=1.01, a_{2}=0.99, A_{s}=0.01, T_{s}=3.1$, and $\sigma_{a}^{2}=3 \times 10^{-6}$.

activator coupling) to $\alpha=0$ (pure inhibitory coupling). The results are illustrated in Fig. 4. We clearly see that increasing the weight of the inhibitory coupling (from right to left) leads to an abrupt suppression of the middle oscillator (Fig. 4 , right) and to a significant joint increase of the linear response $Q$ of the first and third oscillators, but not of the middle one (Fig. 4, left). Note the logarithmic scaling of the parameter $\alpha$ at the abscissa. We clearly observe that already a small fraction of activator coupling (in the order of 1\%) destroys the dynamic trap regime in the given parameter set.

\section{MODEL FOR FOUR NONIDENTICAL UNITS}

Next we delve into the question of whether larger chains with more coupled oscillatory units also show the same phenomenon discussed in the previous section. Although it would seem a rather trivial proposal just to enlarge it to a case in which the system size is $N=4$, the dynamical regimes that arise in such a situation are far from being simple modifications of the results shown above.

We will not consider an enlargement of the excitable ends of the chain, because it is a well known fact that coupling them through the activator variable with a strong enough bind will result in an entrainment of such a subchain and the dynamical evolution of such units will be effectively that of one oscillator. Then, the most interesting question arises

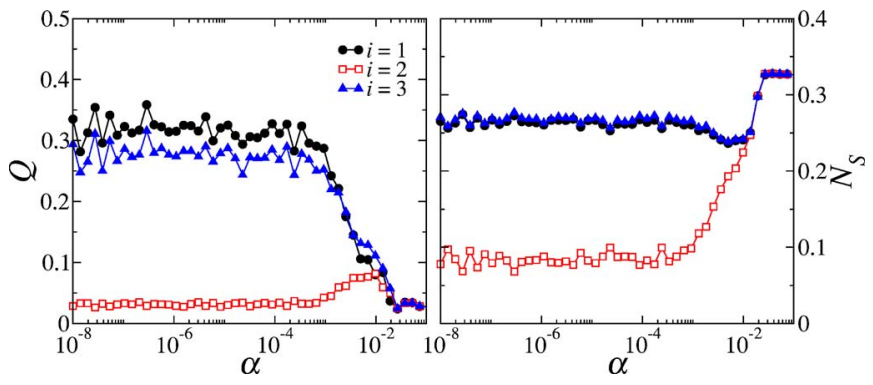

FIG. 4. (Color online) The influence of the type of coupling on the linear response $Q$ (left) and the normalized spike number $N_{S}$ (right) for three coupled FitzHugh-Nagumo units. The sliding parameter $\alpha$ shifts the weight of the diffusion constant $D$ from a pure inhibitory coupling $(\alpha=0.0)$ to a pure activator coupling $(\alpha=1)$. The other parameters are $\varepsilon=10^{-4}, a_{1,3}=1.01, a_{2}=0.99, \sigma_{a}^{2}=4$ $\times 10^{-6}, A_{s}=0.01, T_{s}=3.1$, and $D=0.15$.

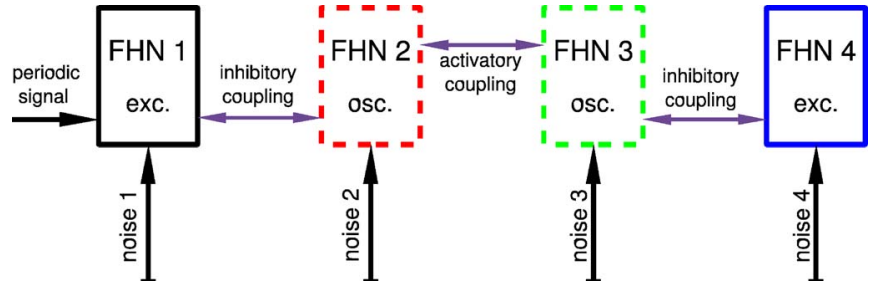

FIG. 5. (Color online) Scheme of the setup for the case $N=4$. While at both ends there are excitable units, in the center there are oscillatory ones. The coupling between units of different nature is inhibitory, and the coupling between the oscillatory units is through the activator variable.

from the enlargement of the middle part, which is composed of oscillatory units. So we add to the scheme of three elements (Fig. 1) an oscillatory element in the middle position and couple it by an activator coupling with the other (identical) oscillatory element and with an inhibitory coupling to the adjacent excitable element (Fig. 5).

The mathematical description of the scheme in Fig. 5 is given in Eqs. (5)-(8). The two oscillatory units are placed at the middle position and are both coupled to their adjacent excitable one by an inhibitory coupling as in the chain of three elements, whereas an activator coupling is set between them. As in the previous section, independent additive white noises act on the units and an external, subthreshold, periodic signal drives only the first element:

$$
\begin{gathered}
\varepsilon \dot{x}_{1}=y_{1}-\frac{x_{1}^{3}}{3}+x_{1}, \\
\dot{y}_{1}=a_{1}-x_{1}+\xi_{1}(t)+A_{s} \sin (\omega t)+D\left(y_{2}-y_{1}\right), \\
\varepsilon \dot{x}_{2}=y_{2}-\frac{x_{2}^{3}}{3}+x_{2}+C\left(x_{3}-x_{2}\right), \\
\dot{y}_{2}=a_{2}-x_{2}+\xi_{2}(t)+D\left(y_{1}-y_{2}\right), \\
\varepsilon \dot{x}_{3}=y_{3}-\frac{x_{3}^{3}}{3}+x_{3}+C\left(x_{2}-x_{3}\right), \\
\dot{y}_{3}=a_{3}-x_{3}+\xi_{3}(t)+D\left(y_{4}-y_{3}\right), \\
\varepsilon \dot{x}_{4}=y_{4}-\frac{x_{4}^{3}}{3}+x_{4}, \\
\dot{y}_{4}=a_{4}-x_{4}+\xi_{4}(t)+D\left(y_{3}-y_{4}\right) .
\end{gathered}
$$

We will fix using the following simulations the parameters $a_{2,3}=0.99$ (oscillatory regime), $a_{1,4}=1.01$ (excitable regime), and the signal intensity $A_{s}=0.01$ (subthreshold).

We are interested in the signal penetration along the chain from the first to the last element as a function of the signal period and the noise intensity. In order to investigate whether the same phenomenon appears in this chain, two different cases are considered: first, we take the optimal parameters from the case $N=3$ and make the coupling between the os- 
cillatory units strong enough such that they become entrained. In the second case, we use a weaker activator coupling.

\section{A. Strong interoscillatory coupling}

Let us focus first on a regime of strong coupling among the oscillatory units. We use the following set of parameters: $\varepsilon=10^{-4}, a_{1,4}=1.01, a_{2,3}=0.99, C=0.80$, and $D=0.22$. In this case and without an external periodic signal $\left(A_{s}=0.0\right)$ injected or noise $\left(\sigma_{a}^{2}=0.0\right)$, the analysis of the power spectrum exhibits that the natural period of the system is $T_{\text {nat }} \approx 2.67$. The oscillatory units exhibit their periodic oscillations at their natural frequency. The excitable units, at their time, show only subthreshold oscillations at the natural frequency of the oscillatory units.

In the presence of an external signal Fig. 6 illustrates the normalized spike number and the linear response $Q$ as a function of the noise intensity $\sigma_{a}^{2}$ for different driving periods $T_{s}$. Figure 6(a) depicts the results when the system is subjected both to noise and external signal and the signal period $T_{s}=2.61$ is slightly below the natural period. It is observed (as in the $N=3$ case) that now the oscillatory units respond but not the excitable one at the end of the chain.

Increasing $T_{s}$ well over the natural frequency, e.g., $T_{s}$ $=2.8$ or 2.9 [Fig. $6(\mathrm{~b})$ or $6(\mathrm{c})$ ], the dynamic trap regime appears. It is important to emphasize that the quality of the signal transmission to the last unit is enhanced with respect to the $N=3$ case (compare Figs. 2 and 6).

An interesting phenomenon occurs for $T_{s}=2.9$ [Fig. 6(c)] where there are two well-differenced situations of dynamic trap like regimes. First, for very low noise intensities $\left(\sigma_{a}^{2}\right.$ $\approx 10^{-7}$ ) there is an almost perfect suppression of the oscillations and at the same time a perfect signal transmission which is the result of the desired dynamic trap regime. There is then a secondary oscillation suppression regime at $\sigma_{a}^{2}$ $\approx 2.5 \times 10^{-6}$, at which the signal is not transmitted with the same fidelity as compared to the case at about $\sigma_{a}^{2} \approx 10^{-7}$. In the second regime the last unit is oscillating, neither at the driving frequency nor at the natural one of the middle oscillators, but at another one. Figure 7 shows the power spectrum for such a secondary regime in the interesting frequency range around driving and resonance frequency. Let us consider the particular case of the fourth oscillator. It is subject to two different signals, one of them with the natural frequency of the third unit and one with the external driving frequency. It is not trivial how these two signals interact in order to produce this unit's response, but it has been demonstrated that in nonlinear systems [30] subjected to two signals, the response may appear at neither any of the driving ones. Regardless of these facts, the important footprint of this secondary regime is the low response of the last unit to the driving frequency.

One can clearly see in Fig. 7 three peaks in the frequency range $\omega \in[1.8,2.8]$ in the system output. The first and highest peak at $\omega \approx 2.16$ is well pronounced only for the first and driven oscillator and corresponds to a period $T \approx 2.9$, equal to the driving period $T_{s}$; i.e., only the driven oscillator exhibits a good response to the signal. The second peak, very close
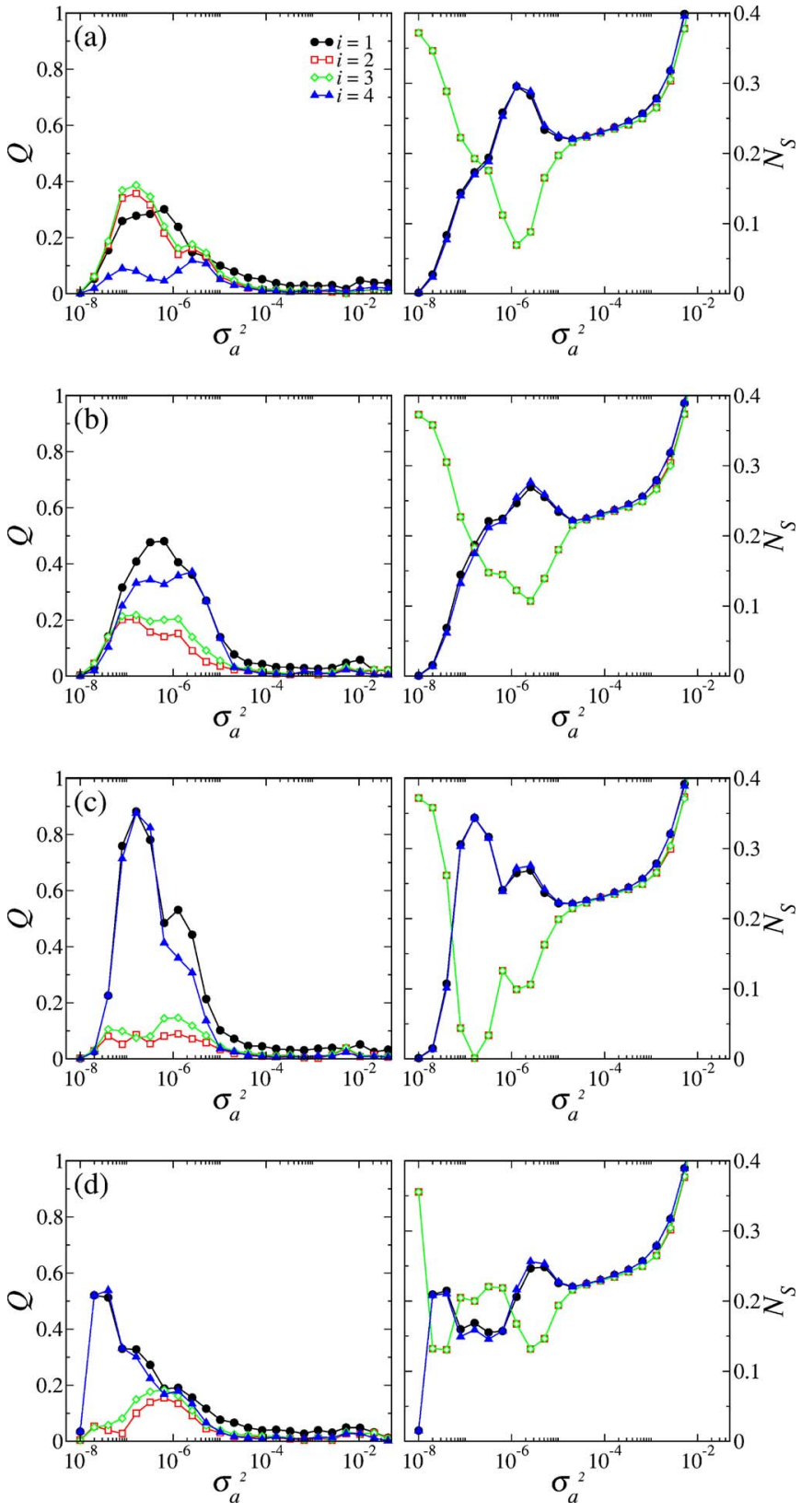

FIG. 6. (Color online) Linear response $Q$ (left column) and $N_{s}$ (normalized spike number), in the right column, versus noise intensity for a chain of four oscillators. (a) $T_{s}=2.61$, (b) $T_{s}=2.8$, (c) $T_{s}$ $=2.9$, and (d) $T_{s}=3.1$. Other parameters: $\varepsilon=10^{-4}, a_{1,4}=1.01, a_{2,3}$ $=0.99$, and $A_{s}=0.01$. The couplings are $C=0.80$ and $D=0.22$ (strong interoscillatory coupling regime).

to the first one, at $\omega \approx 2.245(T \approx 2.8)$ is displayed mainly by the last unit. The third peak at $\omega \approx 2.49(T \approx 2.52)$ can be found in all elements with nearly equal height. The third peak has a very small influence on the total responses of the system (note the logarithmic scale), and it is produced by small subthreshold oscillations. The corresponding time series is as follows: the first (driven) oscillator shows a reliable spiking behavior with a period equal to the driving period, while the two middle oscillators are mostly silent and the last oscillator spikes with a slightly reduced period of $T_{s}=2.8$ (thus leading to the difference in the linear response at the 


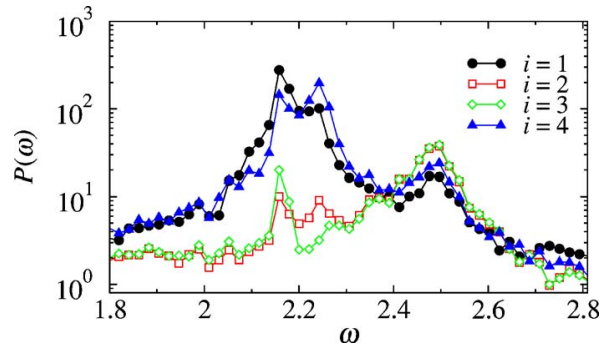

FIG. 7. (Color online) Power spectrum of a four oscillator system for the parameters: $\varepsilon=10^{-4}, a_{1,4}=1.01, a_{2,3}=0.99, A_{s}=0.01$, $T_{s}=2.9, C=0.80, D=0.22$, and $\sigma_{a}^{2}=2.56 \times 10^{-6}$.

signal frequency $Q$ between the first and last FitzHughNagumo in this regime). Due to the small difference in the periodicity, there is no phase locking in this regime and a continuous phase slip between the first and last units appears. If the phase difference is large enough, the chain switches to an antiphase regime; i.e., the otherwise silent middle oscillators spike in antiphase to their excitable neighbors. This transition to the antiphase attractor induces a delay of the last unit compared to the first ones. This antiphase regime is unstable at the considered parameter set, and the chain switches back to the previous attractor with the silent middle elements and the phase slip between the first and last one. The interruption of this long-life attractor by the unstable antiphase attractor results in a nearly equal spike number of the first and last units. Therefore, the interesting behavior in Fig. $6(\mathrm{c})$ at a noise intensity $\sigma_{a}^{2} \approx 2.5 \times 10^{-6}$ is caused by a regime which is only similar to the dynamic trap regime, but is not exactly the desired dynamic trap and hence does not provide a reliable information transport.

As a summary of this section, it could be said that the dynamic trap regime still occurs, but in a narrower region of the driving period $\left(T_{s} \in[2.8,3.0]\right.$, Fig. 8$)$ than in the case of $N=3\left(T_{s} \in[3.0,3.4]\right)$.

Finally, Fig. 9 yields that there is also a range of inhibitory coupling $D$ such that this phenomenon happens. This resonancelike behavior with respect to the inhibitory coupling strength is caused by the influence of this parameter on the resonance frequency of the dynamic trap regime. This figure shows the existence of a maximum (located at a

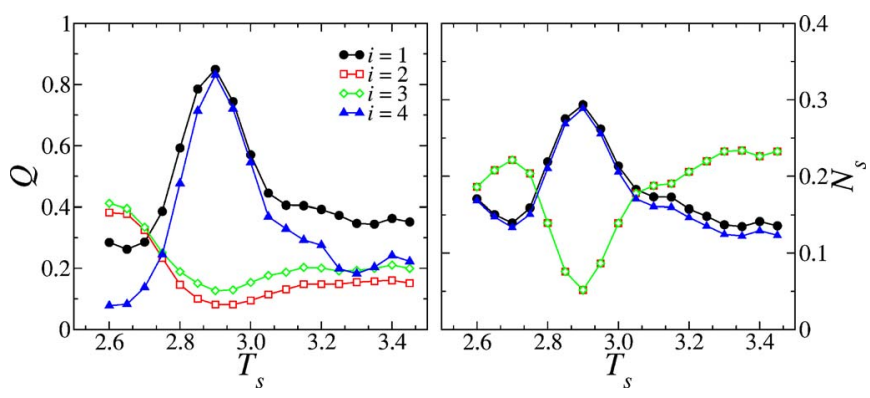

FIG. 8. (Color online) Linear response $Q$ and normalized spike number $N_{s}$ versus time periodicity $T_{s}$. The system is composed of four units in the strong interoscillatory coupling regime and the rest of the parameters are $\varepsilon=10^{-4}, a_{1,4}=1.01, a_{2,3}=0.99, A_{s}=0.01, C$ $=0.80, D=0.22$, and $\sigma_{a}^{2}=2 \times 10^{-7}$.
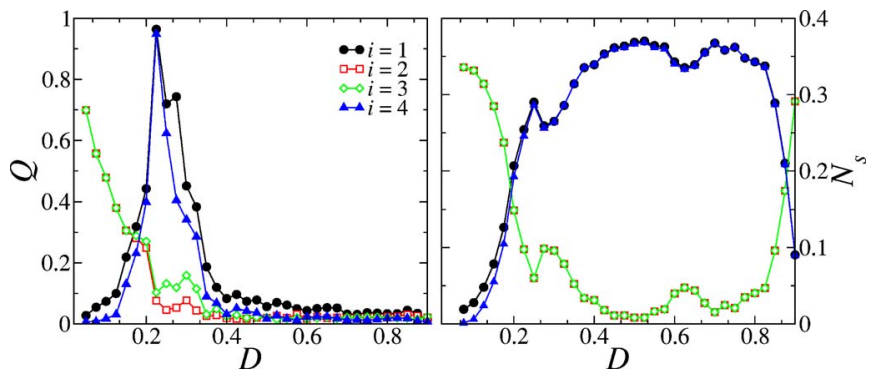

FIG. 9. (Color online) Linear response $Q$ and normalized spike number $N_{s}$ as a function of the inhibitory coupling strength $D$. The system is composed by four units in the strong interoscillatory coupling regime and the rest of the parameters are $\varepsilon=10^{-4}, a_{1,4}=1.01$, $a_{2,3}=0.99, A_{s}=0.01, C=0.80, \sigma_{a}^{2}=2 \times 10^{-7}$, and $T_{s}=2.9$.

coupling $D \approx 0.25$ ) in the response as a function of this parameter.

\section{B. Intermediate interoscillatory coupling}

We also found another kind of dynamic regime in this model with a smaller activator coupling. The analysis of the power spectrum in the absence of noise and external signal shows that the oscillatory units exhibit their periodic oscillations at their natural period $T_{\text {nat }} \approx 2.54$. The excitable units, at their time, generate only subthreshold oscillations at the natural frequency of the oscillatory units. Note that the natural frequency is shifted from the previous case of a strong interoscillatory coupling $\left(T_{n a t} \approx 2.67\right)$. In this case, however, the dependence with $T_{s}$ of the linear response $Q$ curves and the oscillation suppression is quite different from the previous case. Even for slightly detuned input signals $T_{s}=2.55 \mathrm{a}$ strong dynamic trap arises in the system [Fig. 10(a)]. This oscillation suppression mechanism is very robust over a wide range of driving periods $T_{s}$ [Figs. 10(a)-10(c)], whereas a reliable signal transmission along the chain can be observed only in a much narrower range of the driving period, $T_{s}$ $\in[2.6,2.65]$ [Fig. 10(b)].

The oscillation suppression here is really robust, showing that the middle units do not spike for very large periods of time. The phenomenon is also robust to changes of almost four decades in the noise intensity.

Note that the curves for the number of spikes show an exact coincidence between the first and last units (i.e., the excitable ones), although such a perfect matching does not occur for the linear response $Q$. The first and last units fire at the same rate (same normalized spike number $N_{s}$ ), but they are not phase locked; i.e., there is a random phase slip. When the difference in phase between these two excitable units is large enough, this dynamic regime destabilizes and a regime in which there is an in-phase motion of excitable units and (in antiphase) spikes of the oscillatory units appears. But this last dynamic regime is unstable and rapidly falls to the previous one. It is interesting that the matching in the number of spikes occurs in the dynamic trap regime-i.e., that the subthreshold dynamics of the oscillatory units is sufficient to carry information from one end of the chain to the other one.

Figures 11 and 12 demonstrate that there are optimal values of couplings for the suppression to occur. While the de- 

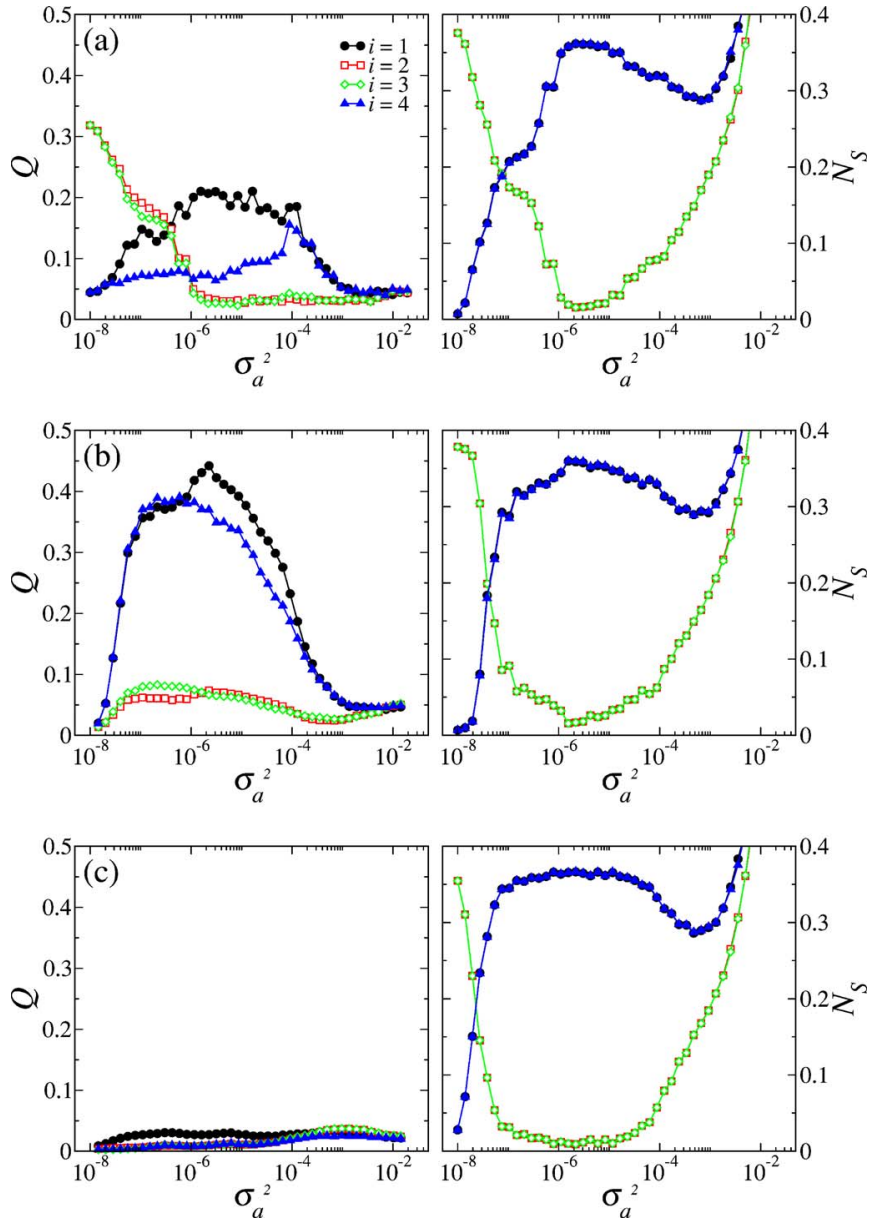

FIG. 10. (Color online) Linear response $Q$ and normalized spike number $N_{s}$ (left and right columns, respectively) versus noise intensity. The time periodicities are (a) $T_{s}=2.55$, (b) $T_{s}=2.61$, and (c) $T_{s}=5.2$. The other parameters are $\varepsilon=10^{-4}, a_{1,4}=1.01, a_{2,3}=0.99$, $A_{s}=0.01, C=0.20$, and $D=0.50$ (intermediate interoscillatory coupling).

pendence on the activator coupling $C$ is such that the suppression holds for couplings larger than a given value, we observe a much narrower range, a resonancelike behavior, as a function of the inhibitor coupling $D$. Even further, for $D$ large enough, the oscillation suppression phenomenon disappears and most of the spikes occur at frequencies different from the driving one (i.e., $Q$ vanishes).
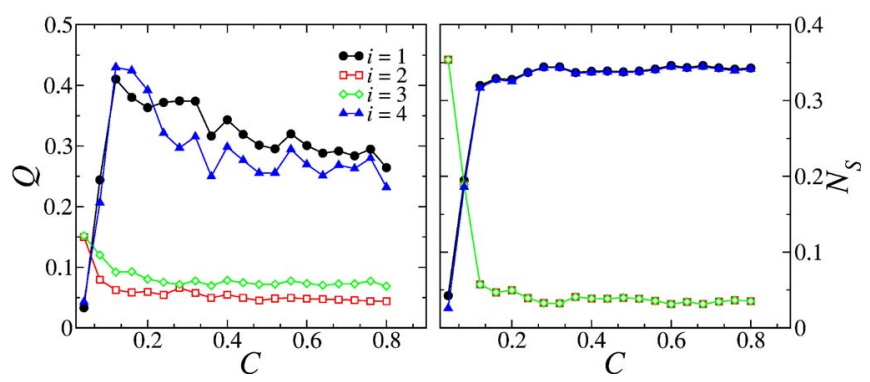

FIG. 11. (Color online) Linear response $Q$ and normalized spike number $N_{s}$ versus activator coupling $C$. The system is composed of four units, and the other parameters are $\varepsilon=10^{-4}, a_{1,4}=1.01, a_{2,3}$ $=0.99, A_{s}=0.01, D=0.50, T_{s}=2.61$, and $\sigma_{a}^{2}=2 \times 10^{-7}$.

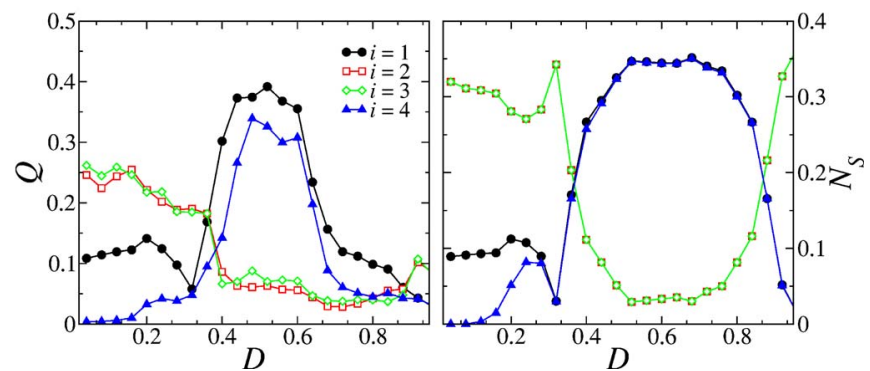

FIG. 12. (Color online) Linear response $Q$ and normalized spike number $N_{s}$ versus inhibitory coupling $D$. The parameters are: $\varepsilon$ $=10^{-4}, a_{1,4}=1.01, a_{2,3}=0.99, A_{s}=0.01, C=0.20, T_{s}=2.61$, and $\sigma_{a}^{2}$ $=2 \times 10^{-7}$.

Figure 13 shows the dependence on the signal periodicity $T_{s}$. It is clear that the oscillation suppression and signal transmission are optimal at the same parameter values. Furthermore, in the same figure it can be seen that there is a very narrow peak around the natural period $\left(T_{s}=T_{n a t}=2.54\right)$ of the oscillatory units at which they respond optimally. Note that the oscillation suppression holds for a wide range of values of the driving period $T_{s}$. But the main result shown in this figure is the fact that the suppression of oscillations in the oscillatory units is much more robust than in the previous cases-i.e., $N=3$ and $N=4$ with strong interoscillatory coupling. This result is somewhat unexpected given the fact that these couplings are not as strong as in the previous parameter sets and then the units are allowed to move more freely.

To show the different influence of activator and inhibitory couplings, we have added an extra activator coupling in the model by interchanging $D$ by $(1-\alpha) D$ in the equations for the inhibitory variable $y_{i}$ and inserting the terms $\alpha D\left(x_{2}-x_{1}\right)$ in Eq. (5), $\alpha D\left(x_{1}-x_{2}\right)$ in Eq. (6), $\alpha D\left(x_{4}-x_{3}\right)$ in Eq. (7), and $\alpha D\left(x_{3}-x_{4}\right)$ in Eq. (8). These extensions of the model are used only in this section for the calculation of Fig. 14. We shift the balance between the activator and inhibitory coupling between these elements continuously with the parameter $\alpha$. In Fig. 14, two clearly different regimes can be observed: for $\alpha<3 \times 10^{-3}$ there is a situation of dynamic trap regime with reliable information transport, while for $\alpha$ $>3 \times 10^{-3}$, there are oscillations in the middle units' position and no response to the driving frequency.

As shown in Fig. 14, a very sharp transition to a situation of oscillation suppression and no response to the driving fre-

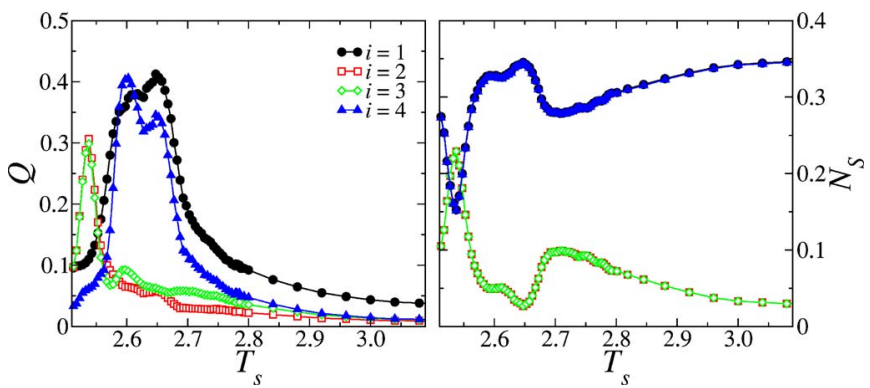

FIG. 13. (Color online) Linear response $Q$ and normalized spike number $N_{s}$ versus driving period $T_{s}$. For this four unit system, the parameters are $\varepsilon=10^{-4}, a_{1,4}=1.01, a_{2,3}=0.99, A_{s}=0.01, C=0.20$, $D=0.50$, and $\sigma_{a}^{2}=2 \times 10^{-7}$. 


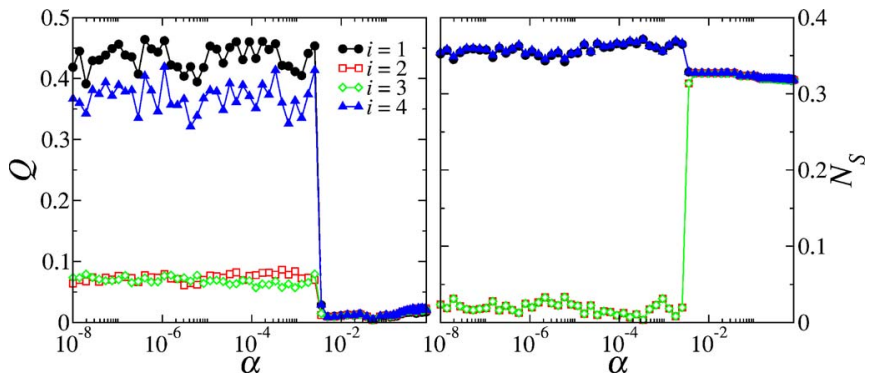

FIG. 14. (Color online) The influence of the type of coupling on the the linear response $Q$ and the normalized spike number $N_{s}$ in a system composed of four coupled FitzHugh-Nagumo units. The sliding parameter $\alpha$ shifts the weight of the diffusion constant $D$ from a pure inhibitory coupling $(\alpha=0.0)$ to a pure activator coupling $(\alpha=1)$. The other parameters are $\varepsilon=10^{-4}, a_{1,4}=1.01, a_{2,3}$ $=0.99, \sigma_{a}^{2}=2 \times 10^{-6}, A_{s}=0.01, T_{s}=2.61, C=0.20$, and $D=0.50$.

quency in the middle units is observed when the activator coupling is weak enough, $\alpha<3 \times 10^{-3}$. Figure 14 (as Fig. 4 for the $N=3$ case) shows the essential imperative of the inhibitory coupling between the excitable and oscillatory units to reach the dynamic trap regime with the desired feature of oscillation suppression and information transmission.

\section{CONCLUSIONS}

In the present work we have studied chains of three or four coupled FitzHugh-Nagumo units subject to noise and to an external signal. The number of units has been chosen in order to keep the number of parameters small, but our model could be exemplary also for larger systems if one regards one oscillator in the model as a representation of a cluster of many oscillators in a close area with similar properties. Conversely, due to the high signal quality at the end of the chain, if we consider replicas of this basic setup coupled linearly to others, the same phenomenon should hold along this chain.

We have found mechanisms which, with the help of a constructive role of the noise, help to suppress self-sustained oscillations in chains of excitable systems while yet allowing for the propagation of external stimuli. In our scheme, an inhibitory coupling between oscillatory and excitable units is essential to reach the dynamic trap regime which is responsible for the oscillation suppression and the information transport. This dynamic trap regime is characterized by an antiphase spiking behavior (with the same frequency of the external signal) of the excitable units at both ends of the chain and a silent (oscillation-suppressed) behavior of the originally oscillatory units in between. The desired dynamic trap regime is sensitive with respect to the driving frequency, the noise intensity, and the coupling strength. We have found other attractors which also offer a reliable oscillation suppression but, however, do not provide a good information transport along the chain. It is interesting to note that the oscillation suppression can also be achieved (in the absence of noise) in the presence of a strong enough driving force. Further study would be needed in order to determine the main features of this suppression of oscillations by the injection of a nonperiodic external signal.
We have considered only paradigmatic models in a very general framework, but we expect that our results are also relevant to other models with inhibitory coupling, used, for example, to describe various physical [31], electronic [16], chemical [20-22,32,33] systems, biological systems [23], including spatial nonuniformities [34], animal coat pattern formation [35], or artificial gene network synchronization with slow autoinducer diffusion [36,37]. An important example is that of the calcium-signaling mechanism [38,39] present in neurons in the thalamus, pancreatic acinar cells, etc. It occurs in regions where the $\mathrm{Ca}^{2+}$ (often responsible for intracell communication) is across the cell membrane to form what is called a $\mathrm{Ca}^{2+}$ wave. Another example is that of the neuronglion interaction $[40,41]$ in which the glion acts in some circumstances as an intermediary messenger between preand post-synaptic neurons. Interestingly enough, most of the models of this interaction are rather simplistic circuits with three or four compartments connected diffusively at first approximation, very close to our own approach.

A similar architecture to the studied here may be responsible, for example, for the activities of neural circuits in a nucleus found in the brain of songbirds [42]. In such circuits the connection between different functional units of the brain is mainly due to inhibitory coupling, whereas the connections within each unit are mainly through the activator variables. Since a strong activator coupling tends to synchronize the population of interacting units, one can neglect, as a first approximation, that each functional unit is composed itself of several units and restrict oneself to a case in which only one (mean) unit is considered for each region of the brain, coupled with others through the inhibitor variable. In this architecture the oscillating element is directly surrounded by inhibitory coupled excitable elements.

The suppression of global oscillations and the prevention of undesirable neural synchronization is an ongoing issue in medicine and neuroscience, and many techniques have been proposed previously in the literature-e.g., permanent highfrequency stimulation $[43,44]$, demand-controlled deep brain stimulation techniques [45,46], delay feedback control of collective synchrony [47] or noise-induced excitability [48]. The results of the present paper can be also potentially useful for this research direction if applied not to inhibitory but negative coupling which can also lead to the appearance of multirhythmicity [49]. There, our result should imply that it is possible to suppress undesirable oscillations while still being able to propagate external stimuli.

\section{ACKNOWLEDGMENTS}

A.Z. acknowledges financial support from VolkswagenStiftung (Germany). C.J.T. wants to thank the Arbeitsgruppe Nichtlineare Dynamik at Universität Potsdam for the kind hospitality extended to him. We acknowledge financial support from the Spanish Government and FEDER (EU) through projects FIS2004-5073, FIS2004-953, and the EU NoE BioSim (LSHB-CT-2004-005137). 
[1] B. Lindner, J. García-Ojalvo, A. Neiman, and L. SchimanskyGeier, Phys. Rep. 392, 321 (2004).

[2] H. Gang, H. Haken, and X. Fagen, Phys. Rev. Lett. 77, 1925 (1996).

[3] A. S. Pikovsky and J. Kurths, Phys. Rev. Lett. 78, 775 (1997).

[4] A. Zaikin, J. García-Ojalvo, R. Báscones, E. Ullner, and J. Kurths, Phys. Rev. Lett. 90, 030601 (2003).

[5] F. Moss, J. Douglass, L. Wilkens, D. Pierson, and E. Pantazelou, Ann. N.Y. Acad. Sci. 706, 26 (1993).

[6] K. Wiesenfeld, D. Pierson, E. Pantazelou, C. Dames, and F. Moss, Phys. Rev. Lett. 72, 2125 (1994).

[7] V. S. Anishchenko, A. B. Neiman, F. Moss, and L. Schimansky-Geier, Phys. Usp. 42, 7 (1999).

[8] A. Pikovsky, M. Rosenblum, and J. Kurths, Synchronization: A Universal Concept in Nonlinear Sciences (Cambridge University Press, Cambridge, England, 2001).

[9] E. I. Volkov and M. N. Stolyarov, Phys. Lett. A 159, 61 (1991).

[10] E. I. Volkov and M. N. Stolyarov, Biol. Cybern. 71, 451 (1994)

[11] S. K. Han, C. Kurrer, and Y. Kuramoto, Phys. Rev. Lett. 75, 3190 (1995).

[12] D. Postnov, S. K. Han, and H. Kook, Phys. Rev. E 60, 2799 (1999).

[13] G. Balázsi, A. Cornell-Bell, A. B. Neiman, and F. Moss, Phys. Rev. E 64, 041912 (2001).

[14] A. Sherman and J. Rinzel, Proc. Natl. Acad. Sci. U.S.A. 89, 2471 (1992).

[15] G. S. Cymbalyuk, E. V. Nikolaev, and R. M. Borisyuk, Biol. Cybern. 71, 153 (1994).

[16] D. Ruwisch, M. Bode, D. Volkov, and E. Volkov, Int. J. Bifurcation Chaos Appl. Sci. Eng. 9, 1969 (1999).

[17] E. I. Volkov and D. V. Volkov, Phys. Rev. E 65, 046232 (2002).

[18] E. I. Volkov, E. Ullner, A. A. Zaikin, and J. Kurths, Phys. Rev. E 68, 061112 (2003).

[19] E. Volkov, E. Ullner, and J. Kurths, Chaos 15, 023105 (2005).

[20] V. K. Vanag, A. M. Zhabotinsky, and I. R. Epstein, J. Phys. Chem. A 104, 11566 (2000).

[21] W. Wang, I. Z. Kiss, and J. L. Hudson, Phys. Rev. Lett. 86, 4954 (2001).

[22] S. Nakata, T. Miyata, N. Ojima, and K. Yoshikawa, Physica D 115, 313 (1998).

[23] D. T. W. Chik, S. Coombes, and Z. D. Wang, Phys. Rev. E 70, 011908 (2004).

[24] R. A. FitzHugh, Biophys. J. 1, 445 (1961).

[25] J. P. Keener and J. Sneyd, Mathematical Physiology (Springer,
New York, 1998).

[26] Most of our results come from a numerical integration of the previous equations using a stochastic Runge-Kutta-type method known as the Heun algorithm.

[27] J. García-Ojalvo and J. M. Sancho, Noise in Spatially Extended Systems (Springer, New York, 1999).

[28] L. Gammaitoni, P. Hänggi, P. Jung, and F. Marchesoni, Rev. Mod. Phys. 70, 223 (1998).

[29] P. Landa and P. McClintock, J. Phys. A 33, L433 (2000).

[30] D. R. Chialvo, O. Calvo, D. L. Gonzalez, O. Piro, and G. V. Savino, Phys. Rev. E 65, 050902(R) (2002).

[31] B. Kerner and V. Osipov, Sov. Phys. Usp. 33, 679 (1990).

[32] V. Vanag, L. Yang, M. Dolnik, A. Zhabotinky, and I. Epstein, Nature (London) 406, 389 (2000).

[33] V. Castets, E. Dulos, J. Boissonade, and P. De Kepper, Phys. Rev. Lett. 64, 2953 (1990).

[34] G. Balázsi, A. H. Cornell-Bell, and F. Moss, Chaos 13, 515 (2003).

[35] H. Meinhardt, Models of Biological Pattern Formation (Academic Press, New York, 1982).

[36] D. McMillen, N. Kopell, J. Hasty, and J. J. Collins, Proc. Natl. Acad. Sci. U.S.A. 99, 679 (2002).

[37] A. Kuznetsov, M. Kærn, and N. Kopell, SIAM J. Appl. Math. 65, 392 (2004).

[38] X. Wang, J. Rinzel, and M. Rogawski, J. Neurophysiol. 66, 839 (1991).

[39] G. Ullah, P. Jung, and A. H. Cornell-Bell, Cell Calcium 39, 197 (2006).

[40] D. Postnov, L. Ryazanova, E. Mosekilde, and O. Sosnovtseva, Int. J. Neural Syst. 16, 99 (2006).

[41] D. Postnov, L. Ryazanova, and O. Sosnovtseva, J. Neutral Systems, 16, 99 (2006).

[42] R. Laje and G. B. Mindlin, Phys. Rev. Lett. 89, 288102 (2002).

[43] A. Benabid, P. Pollack, and C. Gervason, Lancet 337, 403 (1991).

[44] S. Blond et al., J. Neurosurg. 77, 62 (1992).

[45] P. Tass, Phase Resetting in Medicine and Biology-Stochastic Modelling and Data Analysis (Springer, Berlin, 1999).

[46] P. Tass, Europhys. Lett. 53, 15 (2001).

[47] M. G. Rosenblum and A. S. Pikovsky, Phys. Rev. Lett. 92, 114102 (2004).

[48] E. Ullner, A. Zaikin, J. García-Ojalvo, and J. Kurths, Phys. Rev. Lett. 91, 180601 (2003).

[49] P. Dayan and L. F. Abbott, Theoretical Neuroscience: Computational and Mathematical Modeling of Neural Systems (MIT Press, Cambridge, MA, 2001). 